\title{
Peace from Home to School for Children with Attention-Deficit and Hyperactivity Disorder (ADHD)
}

\author{
Ayoka Mopelola Olusakin and Mopelola Omoegun \\ Department of Educational Foundations, Faculty of Education, University of Lagos, \\ Akoka-Lagos, Nigeria
}

KEYWORDS Peace; home; school; parents of children with ADHD; motivation; behavior modification skills
training

ABSTRACT This study examines the impact of behavior modification skills training for parents of children with ADHD on their level of involvement in motivating such children to learn peacefully. 27 of such parents (all mothers) whose children's ages range from 7 to 10 years with a mean age of 9 years, signed up for the program. The statistical analyses of their self-rating before and after the training, showed a significant difference in their efforts at motivating their children with ADHD to learn to be at peace. The results revealed that they benefited from the program. The counseling implications were discussed.

\section{INTRODUCTION}

Most people want peace of mind in their home, neighborhood, school, workplace, and the society. The UNESCO in its effort at promoting the Culture of Peace developed what it tagged 'the Manifesto 2000' which declares: “Aware of my portion of responsibility I (...) make the commitment in my daily life, my family, my work, my community, my country, my region to: respect all lives, reject violence, liberate my generosity, listen to understand, preserve the planet, reinvent solidarity"

The main message of the manifesto 2000 is the call to personal responsibility. This millennium should usher human race into a new beginning, an opportunity to turn, all together the culture of war and violence into a culture of peace and non-violence. According to Oliner et al. (1991), peace is much more than just the absence of war. Everyone has a responsibility towards the promotion of the culture of peace. Hence, it is important that children with or without ADHD be reached through their parents to be at peace.

Attention Deficit Hyperactivity Disorder (ADHD) is one of the most common mental disorders that develop in children and affects not only their peace but that of their parents, siblings, teachers and peers. According to Barkley

Address for Correspondence: Dr. Ayoka Mopelola Olusakin, Department of Educational Foundations, Faculty of Education, University of Lagos,

Akoka-Lagos, Nigeria.

E-mail: mopeolusakin@yahoo.com
(1990), as a globally detected disorder, Attention Deficit Hyperactivity Disorder (ADHD) is the diagnostic label given to children presenting significant problems with attention, impulse control, and activity levels. ADHD is not a newly discovered disorder, been in medical literature for over a hundred years. What is new however, is the increased awareness of the disorder (Silver, 1999). Children with ADHD have impaired functioning in multiple settings, including home, school, and in relationships. If left unattended to, the disorder can have long-term adverse effects into adolescence and adulthood.

Symptoms of ADHD usually appear over the course of many months. Even though these symptoms appear early in a child's life but because most children are sometimes restless, sometimes act without thinking, and sometimes daydream the time away, ADHD is not easy to diagnose. Also because many normal children may have these symptoms, but at a low level, and the symptoms may be caused by another disorder. Different symptoms may appear in different settings, depending on the demands the situation may pose for the child's self-control. A child who finds it difficult to sit still or is otherwise disruptive is more likely to be noticeable in school, while the inattentive daydreamer may be overlooked. The impulsive child who acts before thinking may be considered just a "discipline problem," while the child who is passive or sluggish may be viewed as merely unmotivated. Yet both may have different types of ADHD. When the child's hyperactivity, distractibility, poor concentration, or impulsivity begin to affect performance in 
school, social relationships with other children, or behavior at home then that child may have to go through ADHD diagnosis.

If ADHD is suspected, it is important that the child receive a thorough examination and appropriate diagnosis by well-qualified professionals with training in ADHD. This includes child psychiatrists, psychologists, developmental/ behavioral pediatricians, behavioral neurologists, and clinical social workers. After ruling out other possible reasons for the child's behavior, the specialist checks the child's school and medical records and talks to teachers and parents who have filled out a behavior rating scale for the child. A diagnosis is made only after all the information has been considered

Symptoms of ADHD will appear over the course of many months, often with the symptoms of impulsiveness and hyperactivity preceding those of inattention, which may not emerge for a year or more.

According to the American Psychiatric Association (1994) version of the Diagnostic and Statistical Manual of Mental Disorders (DSMIV), which is the main diagnostic reference of the Mental Health Professionals in the United States of America and also adopted in Nigeria, there are three patterns of behavior that indicate ADHD. People with ADHD may show several signs of being consistently inattentive. They may have a pattern of being hyperactive and impulsive far more than others of their age. Or they may show all three types of behavior. There are three subtypes of ADHD recognized by professionals. These are the predominantly hyperactiveimpulsive type (that does not show significant inattention); the predominantly inattentive type (that does not show significant hyperactiveimpulsive behavior) sometimes called ADD; and the combined type (that displays both inattentive and hyperactive-impulsive symptoms).

Some of the signs of ADHD as highlighted in DSM-IV out of which 6 (or more) should have persisted for at least 6 months to a degree that is maladaptive and inconsistent with the developmental level are:

\section{Inattention}

often fails to give close attention to details or makes careless mistakes in schoolwork, work, or other activities:

- often has difficulty sustaining attention in tasks or play activities;

often does not seem to listen when spoken to directly;

often does not follow through on instructions and fails to finish schoolwork, chores, or duties in the workplace (not due to oppositional behavior or failure to understand instructions);

often has difficulty organizing tasks and activities;

- often avoids, dislikes, or is reluctant to engage in tasks that require sustained mental effort (such as schoolwork or homework);

- often loses things necessary for tasks or activities (e.g., toys, school assignments, pencils, books, or tools);

- is often easily distracted by extraneous stimuli;

is often forgetful in daily activities

\section{Hyperactivity}

- often fidgets with hands or feet or squirms in seat ;

- often leaves seat in classroom or in other situations in which remaining seated is expected;

- often runs about or climbs excessively in situations in which it is inappropriate (in adolescents or adults, may be limited to subjective feelings of restlessness); often has difficulty playing or engaging in leisure activities quietly;

- is often "on the go" or often acts as if "driven by a motor";

often talks excessively

\section{Impulsivity}

- often blurts out answers before questions have been completed;

- often has difficulty awaiting turn;

- often interrupts or intrudes on others (e.g., butts into conversations or games)

It was also noted that some hyperactiveimpulsive or inattentive symptom that caused impairment were present before the age of 7 years.

To assess whether a child really has ADHD, specialists consider several critical questions: Are these behaviors excessive, long-term, and pervasive? That is, do they occur more often than in other children the same age? Are they a continuous problem, not just a response to a 
temporary situation? Do the behaviors occur in several settings or only in one specific place like the playground or in the schoolroom?

Because most children show some of these behaviors at times, the diagnosis requires that such behavior be demonstrated to a degree that is inappropriate for the person's age. The diagnostic guidelines also contain specific requirements for determining when the symptoms indicate ADHD. The behaviors must create a real handicap in at least two areas of a person's life such at home, in the schoolroom, on the playground, in the community, or in social settings. So someone who shows some symptoms but whose schoolwork or friendships are not impaired by these behaviors may not be diagnosed with ADHD. Nor would a child who seems overly active on the playground but functions well elsewhere be confirmed as having an ADHD.

\section{Secondary Symptoms Common in Children with ADHD}

Although the major symptoms of ADHD include inattention, hyperactivity, and impulsiveness, there are many other areas in a child's life could be affected by a diagnosis of ADHD such as:

Difficulty in Controlling Emotions: Many times children with ADHD have a hard time controlling their emotions. This may be caused, not by anger, but by over-stimulation. Children with ADHD have a difficult time sorting out all the stimulation in their lives as they pay attention to everything around them. When they become over-stimulated, their emotions may not be able to cope and a temper tantrum or emotional outburst might follow.

High Sensitivity: Children with ADHD may have heightened sensory awareness and be highly sensitive to touch, smell and other sensory stimulus. For example they could find it uncomfortable to be touched by other people or to be disturbed by strong smells.

Many People with ADHD Have Difficulty Falling and Staying Asleep. Their thoughts may be racing and they might not able to slow down their minds enough to relax and fall asleep. Additionally, for those that are hyperactivity, movement may not stop just because they are asleep. Tossing and turning throughout the night could be a common complaint of those with ADHD (Silver, 1999).
Children with ADHD Have a Tendency to be Disorganized. This also often causes academic problems, as they can often lose their workbooks, materials or papers that were to be handed in. This can also create problems at home with their clothes, shoes and toys scattered all around the rooms.

Inability to Plan Ahead: Children with ADHD may not be able to associate the future with the current moment. Therefore, they are often procrastinators and lack the ability to plan an event or outing. Parents can help children by using a planning worksheet to help children work out a plan for outings with friends or with long term projects at school.

Short Term Memory might seem to be absent in some children with ADHD because they do not seem to remember from moment to moment what they had done. Children with Short Term Memory problems often have academic difficulties. They may have problems remembering what to do for homework or with handing in the ones they had done. They may forget what they were doing, only moments after being given instructions.

Difficulty Moving from One Activity to Another is another problem area for children with ADHD. As much as they have difficulty focusing on one task, they also can become glued to the ones they are interested in. It can become hard for the child to move away from this task and continue to the next task at hand.

Time Management Problems: Children with ADHD have time management problems because they do not often realize the passage of time. For example, when they have been playing for an hour, they may feel that only about 15 minutes has passed. Along that line, it is hard for them to complete and move onto the next task. Even on a Saturday, when there is no school, children may need help in planning their time. They may want to include several activities in the day, including playing with friends, playing video games. They may have chores to complete at home as well. For a child with ADHD, they could begin one activity and not realize just how much time has passed and then be disappointed there is no time left for other activities. Helping them plan their day will provide them with variety and keep them happy throughout the day (Barkley, 2000).

Not every child with ADHD will exhibit all of the additional characteristics along with their ADHD. Some of the children may also exhibit 
these characteristics with different levels of severity. While one child with ADHD may exhibit short term memory difficulties, he may not have emotional outbursts, while others may have sleep problems others may have time management problems

What are the causes of ADHD? Some of the first questions a parent might ask could be "Why my child? What did I do wrong?”(Wender, 2002). There has also been no connection made between birthing complications, or the manner in which parents raise their children, and the development of ADHD. Most substantiated causes appear to fall in the realm of neurobiology and genetics. This is not to say that environmental factors may not influence the severity of the disorder, and especially the degree of impairment and suffering the child may experience, but that such factors do not seem to give rise to the condition by themselves.

Scientists are studying causes in an effort to identify better ways to treat, and perhaps someday, to prevent ADHD. They are finding more and more evidence that ADHD does not stem from the home environment, but from biological causes. Knowing this can remove a huge burden of guilt from parents who might blame themselves for their child's behavior. Over the last few decades, scientists have come up with possible theories about what causes ADHD. Some of these theories have led to dead ends, some to exciting new avenues of investigation. But according to NHMRC (1997) what causes ADHD is essentially unknown. Scientists and researchers have found it difficult to find a single accepted cause for the disorder.

\section{SUGGESTED CAUSES INCLUDE}

Cigarettes Smoking and Drinking of Alcohol During Pregnancy: Although there is little compelling evidence that ADHD can develop purely from social factors or child-rearing methods, but some studies have shown a possible correlation between the use of cigarettes and alcohol during pregnancy and risk for ADHD in the offspring of that pregnancy. The National Health and Medical Research Council (NHMRC)'s (1997) have reported that maternal substance abuse during pregnancy may be associated with ADHD, where substances such as cocaine and nicotine induce ADHD-like symptoms (Chronis, Lahey, Phelham, Kipp, Baumann, and Lee, 2003).
As a precaution, it is best during pregnancy to refrain from both cigarette and alcohol use.

Another environmental agent that may be associated with a higher risk of ADHD is high levels of lead in the bodies of young preschool children. Since lead is no longer allowed in paint and is usually found only in older buildings, exposure to toxic levels is not as prevalent as it once was. Children who live in old buildings in which lead still exists in the plumbing or in lead paint that has been painted over may be at risk.

Brain Injury: One early theory was that attention disorders were caused by brain injury. Some children who have suffered accidents leading to brain injury may show some signs of behavior similar to that of ADHD, but only a small percentage of children with ADHD have been found to have suffered a traumatic brain injury.

Refined Sugar and Food Additives: Some people are of the opinion that symptoms of ADHD are exacerbated by refined sugar or food additives. In 1982, the American National Institutes of Health held a scientific consensus conference to discuss this issue (Consensus Development Panel, 1982). It was found that diet restrictions helped about 5 percent of children with ADHD, mostly young children who had food allergies. Wolraich, Milich, Stumbo, and Schultz (1985) carried out a study on the effect of sugar on children, using sugar one day and a sugar substitute on alternate days, without parents, staff, or children knowing which substance was being used and reported no significant effects of the sugar on behavior or learning.

In another study by Hoover and Milich (1994), children whose mothers felt they were sugarsensitive were given aspartame as a substitute for sugar. Half the mothers were told their children were given sugar, half that their children were given aspartame. The mothers who thought their children had received sugar rated them as more hyperactive than the other children and were more critical of their behavior.

Genetics: Wodrich (2000) has summarized that ADHD is at least partially inherited, and not generally caused by brain injury or external factors like sugar ingestion. Attention disorders often run in families, so there are likely to be genetic influences. Studies indicate that 25 percent of the close relatives in the families of ADHD children also have ADHD, whereas the rate is about 5 percent in the general population. Many studies of twins now show that a strong 
genetic influence exists in the disorder (Biederman et al., 1990).

Researchers continue to study the genetic contribution to ADHD and to identify the genes that cause a person to be susceptible to ADHD. Since its inception in 1999, the Attention-Deficit Hyperactivity Disorder Molecular Genetics Network has served as a way for researchers to share findings regarding possible genetic influences on ADHD. Giler (2000) proposes that ADHD appears to be mainly the result of neurotransmitter deficiency in a specific area of the brain, more specifically the frontal cortex region, causing an absence in the ability to control certain behaviors

Medical Treatment: Treatment through medication is of utmost importance. This is because it is believed that ADHD is caused primarily by a deficiency of a specific neuro-transmitter, the goal of medication is to increase the level of the neurotransmitter involved (Silver, 1999). The most widely recommended medication for the disorder is stimulants such as Methylphendate and Dextroamphetamine. According to Barkley (1990), these stimulants, work by primarily increasing how much of these chemicals are available in the brain, the stimulants increase the action of these brain cells, which seem to be responsible for inhibiting the behavior and helping to stick to what is being done.

There was a report in year 2002, that the researchers in the NIMH Child Psychiatry Branch had studied 152 boys and girls with ADHD, matched with 139 age- and gender-matched controls without ADHD. The children were scanned at least twice, some as many as four times over a decade. As a group, the ADHD children showed 3-4 percent smaller brain volumes in all regions - the frontal lobes, temporal gray matter, caudate nucleus, and cerebellum.

This study also showed that the ADHD children who were on medication had a white matter volume that did not differ from that of controls. Those never-medicated patients had an abnormally small volume of white matter. The white matter consists of fibers that establish longdistance connections between brain regions. It normally thickens as a child grows older and the brain matures.

Although this long-term study used MRI to scan the children's brains, the researchers stressed that MRI remains a research tool and cannot be used to diagnose ADHD in any given child.
Children with ADHD taking stimulants are found to be less impulsive, and have fewer problems with aggression, noisiness, noncompliance, and disruptiveness.

However, with the use of these stimulants, Silver (1999) has reported symptoms of anxiety, tension, agitation, loss of appetite, and insomnia.

Because of the uncertainty of ADHD's origin, there is also a cloud of uncertainty regarding its treatment. Silver (1999) proposes that the treatment of ADHD should involve a multi-disciplinary approach. Thus, individual and family education, counseling with appropriate behavioral management programs, and appropriate medications need to be integrated.

\section{The Need for Motivation}

Motivation which is often defined as a need or drive that energizes behavior toward a goal is the key to success. Children turn to parents, teachers and peers to discover the "why" of learning. Parents are central to their children's motivation. One of the most common problems that parents and teachers face is lack of motivation on the part of the children. Motivation can either come from within the student (intrinsic) or from outside (extrinsic). A child who is intrinsically motivated performs a task because of the joy that comes from learning new materials. A child who performs in school to gain parent approval, grades, or rewards is externally motivated. While research shows that those children with internal motivation may achieve greater success, teachers and parents often find that many children seek external reinforcements (Wender, 2002). Parents who ask questions that lead to more questions for a child are more successful in developing intrinsic motivation. Motivation often varies depending on the setting, the people involved, the task and the situation. The key to helping each learner is to find that which motivates him.

\section{Purpose of Study}

There is a dearth of information on ADHD and the various forms of treatment among Nigerians compared to Polio and malaria. This study is aimed at training some Nigerian parents in some behavior modification skills to help their ADHD children to learn peacefully. In effect the study sought answer to the research question of whether or not there would be any significant 
difference in the participants self-rating on each of the behavior modification skills they are exposed to in this study before and after the training.

Wodrich (2000) states that behavior modification's most basic idea is that children learn many of their behaviors from their environment, and so, children with ADHD, although with impulse control and attention problems, also learn many behaviors from the world around them. It is important then for parents to work with their children, health professionals, family members, school teachers, and the like to re-teach more acceptable behaviors.

Research on parenting and ADHD has revealed that ADHD has important consequences to the sufferers, as well as their parents and siblings (Swensen, Birnbaum, Secnik, Marynchenko, Greenberg, and Claxton, (2003). More specifically there has been discovery that parents experience greater stress than other parents with children without ADHD, because of the additional parenting challenges they face (Podolski and Nigg, 2001).

According to Barkley (2000), parents of children with ADHD move through several steps in their efforts to control their children's disruptive behavior. Initially, parents try to ignore or withhold attention from their children when the child demonstrates disruptive behavior. In doing this, parents believe that the disruptive behavior is intended merely to get attention, and so believe that ignoring it will decrease the problem. But, such behavior from a child with ADHD is not the result of attempts for attention, and thus ignoring the behavior may not produce the required result. As the behavior continues or intensifies, parents move on to give more commands and directives, especially those aimed at controlling the child's impulses. These commands are often restrictive requiring them to stop what they are doing, and parents will find themselves repeating them frequently at times with annoyance and frustration. And, when this approach fails to motivate the child with ADHD to listen and obey, parents may move to the use of punishment, or loss of privileges and even physical discipline.

\section{METHOD}

The setting of this study was the Faculty of Education, University of Lagos, Nigeria. Interested parents of children with ADHD who came for counseling were given the opportunity to sign up for a 7-week behavior modification training program.

27 parents (all mothers) whose ADHD children aged between 7 and 10 years with a mean age of 9 years, signed up for the program. These mothers were within the age range of $31-52$ years, with a mean age of 39.4 years.

All the 27 parents who signed up for the behavior modification skills training program formed the participants in this study. These participants were exposed to the program which was conducted in 7 sessions. Each of the sessions lasted for about 2 hours per week (the time chosen by $92 \%$ of the participants and eventually agreed upon by all the participants).

During the $1^{\text {st }}$ session, each of the participants was asked to rate herself on each of the 10 Behavior Modification Skills on a scale of 1-4 (Quite unlike $m e=1$, unlike $m e=2$, like $m e=3$, quite like me=4). The data collected formed the pre-test data.

The participants shared their experiences and then they were briefed on the etiology of ADHD for them to have an understanding of their children's behavior. After this they were exposed to 10 behavior modification skills. The training program involved the use of a combination of written materials and verbal instructions in specific skills as follow:

1) Relaxation as a Parent: Relax to reduce your stress level and to increase your own tolerance for frustration so that you can respond more peacefully to your child's behavior. You have to manage your own health and well-being while raising a child with ADHD. As a parent, your focus should be on looking forward and finding the best possible way to help your ADHD child and not to engage in self-blame or self-condemnation. You should know

- what to expect from a child with ADHD;

- effective, non-confrontational, communication with your child;

- consistent and practical time management;

- how to utilize the available community resources, support groups and make use of online resources that can fill the gap between the support provided by face-to-face community resources

2) Identification of Specific Desirable Behavior: Identify specific desirable behaviors that you want to encourage in the child: It is necessary for the parent to set specific goals by identifying the desired behaviors. For example, 
asking for a toy instead of grabbing it, or completing a simple task rather than moving aimlessly around the house. The goal, over time, should be to help children learn to control their own behavior and to choose the more acceptable behaviors.

3) Structuring of Situation For Child's Success: Structure situations in ways that will allow the child to succeed. This may include allowing only one or two playmates at a time, so that the child doe not get over stimulated. Or if their child has trouble completing tasks, they may learn to help that child divide a large task into small steps, and then praise the child as each step is completed. Regardless of the specific technique a parent may use to modify the child's behavior, some general principles appear to be useful for most children with ADHD. These include providing more frequent and immediate feedback (including rewards and punishment), setting up more structure in advance of potential problem situations, and providing greater supervision and encouragement to children with ADHD in relatively hard situations. Relaxation as a parent

4) Have a Schedule of Daily Activities: Children with ADHD may need help in organizing their time. Have the same routine every day, from wake-up time to bedtime. The schedule should include homework time and playtime (including outdoor recreation and indoor activities such as computer games). Have the schedule on the refrigerator or a bulletin board in the kitchen. If a schedule change must be made, make it as far in advance as possible.

Useful everyday items should be organized. Have a place for everything and keep everything in its place. This includes clothing, and school supplies.

Use homework and notebook organizer. Emphasize the importance of writing down assignments and bringing home needed books.

5) Decision on a System of Reward: Decide on a system of reward: The child should be told exactly what is expected in order to earn the reward and he receives the reward when he performs the desired behavior and a mild penalty when he does not. A reward can be small, perhaps a token that can be exchanged for special privileges, but it should be something the child wants and is eager to earn. Token or point systems could be used to immediately reward a desirable behavior. Rewards do not always need to be money; At times include it could be a tangible reward for an extended period of time of compliance. Smaller goals may have rewards such as stickers, stars, or just verbal commendation. Give rewards often, the more intense the behavior was at the beginning, the more often the rewards should come. Feedback could be provided on attitude many times each day. Providing immediate feedback and rewards/ consequences helps to keep a child focused. In addition, children with ADHD tend to have difficulty relating a consequence to an action if they do not occur simultaneously or just shortly after. Because children with ADHD often receive, and expect, criticism, look for good behavior and praise it.

Several times a day, praise your child for doing something right and if necessary, set up the situation for your child to succeed.

6) Having Consequences for Undesirable Behavior: Have certain consequences in place for undesirable behaviors:

Consequences should be delivered peacefully each time to avoid being frustrated over the behavior. Time-out or isolation could be employed when the child becomes too unruly. During timeouts, the child is removed from the agitating situation and sits alone quietly for a short time to calm down.

Many times, once a behavior program has been set up, parents complain that behaviors become worse. This could be normal because the child could have learned to rely on certain reactions. He might have come to understand and to associate such reaction with caring. It is only likely therefore, that he is craving for the security of the old reaction. But by being persistent, the child would begin to perceive the new reaction as the correct one and try to attain the reward.

7) Avoidance of Information Overload: Avoid information overload: Children with ADD/ ADHD tend to get confused easily with multiple information. In order to help them develop better habits and behaviors, there is need to choose one specific behavior to work on at a time. More could be added later.

For example, it could be to stop being aggressive toward siblings, and then stop running in the house. But make sure it is specific. Parents are encouraged to set realistic goals that are attainable. Making use of short term goals helps. Breaking the day down to short intervals can help in creating a desire to succeed. It is much easier for a child to behave for 30 minutes than it is for 
them to behave for an entire day. They are to set their children up for some early successes for which praise should be doted out.

8) Consistency: Be consistent and focus on the target behaviors: Children with ADHD need consistent rules that they can understand and follow. If rules are followed, give small rewards. Provide contingencies that follow behavior quickly and consistently, and incorporate novelty to maintain interest. Without consistency the child might not have the same motivation to act correctly. If the consequences are not consistent the child may still continue to take chance that there might not be any consequence at times.

The reward can be changed without changing the rules because the child could become bored with the same tokens all the time. Rules should remain the same, but changing the delivery pattern can help to keep it fresh.

9) Play/Leisure for the Child: Give the child some time each day, for relaxation/leisure and regular exercise with you as a parent. Use jokes and humor appropriately. Smile and laugh together as you either take a walk in the garden or tell folktales/stories in the living room.

10) Move on to Another Behavior: Once the child has mastered the initial behavior, move on to something else. You should make use of the motivational steps again following a child's interests, when possible, rather than spending time building elaborate reward system.

Participants were encouraged to start putting into practice the behavior modification skills right from the first session of training and they were given the hand-outs containing all the skills during the first session. In rounding up the $7^{\text {th }}$ session of the training, each participant was given the opportunity to rate herself again to collect the post-test scores. The pre- and post-test scores were them subjected to the 'same group T (dependent) test statistical analysis as shown in 'the table showing the result of the T-Test statistics of the Pre- and Post-test scores of the participants in the Behavior Modification Skills'.

\section{RESULTS}

The statistical analyses of the participants self-rating scores on each of the behavior modification skills before and after the training showed significant difference in the efforts of the parents at motivating their ADHD children to learn to be at peace (See the Table showing the result of the t-Test statistics of the Pre- and Post-test scores of the participants in the Behavior Modification Skills) Parents who participated in the training reported higher mean scores after the training compared to their self-rating mean scores before the training. All these higher post-test mean scores (except the one for skill number 10 which has to do with 'moving on to the next behavior') were statistically significant at .05 . Even the one that was not statistically significant still has higher post-test mean which might not be significant because of the shortness of the training period. Some of the parents might not have the need to actually move on to another behavior.

The finding showed that the parents who participated in the study considered themselves to have benefited from it. This was supported by Weinberg (1999) who studied the use of parent training and reported that parent training enhanced knowledge and understanding of ADHD. According to Swensen et al. (2003), research has revealed findings that without relevant parenting skills, parental experience with a child with ADHD lead to mood and anxiety disorders. The findings also support Podolski and

Table 1: $t$-Test statistics of the pre- and post-test scores of the participants in the behavior modification skills

\begin{tabular}{|c|c|c|c|c|c|}
\hline Behavior Modification Skills & $\begin{array}{l}\text { Pre-test } \\
\text { Means }\end{array}$ & $\begin{array}{l}\text { Post-test } \\
\text { Means }\end{array}$ & $\begin{array}{l}\text { Standard } \\
\text { Deviation }\end{array}$ & $\begin{array}{c}T \\
\text { (observed) }\end{array}$ & $\begin{array}{c}\text { Significance } \\
\text { (2-tailed) }\end{array}$ \\
\hline Relaxation as a parent & 1.482 & 2.519 & 1.055 & 5.106 & $.000 *$ \\
\hline Identification of specific desirable behavior & 1.482 & 3.222 & .764 & 11.836 & $.000 *$ \\
\hline Structuring of situation for child's success & 1.741 & 2.889 & 1.231 & 4.846 & $.000 *$ \\
\hline Schedule of daily activities & 1.630 & 3.037 & 1.118 & 6.539 & $.000 *$ \\
\hline Decision on a system of reward & 1.259 & 2.778 & 1.014 & 7.780 & $.000 *$ \\
\hline Having consequences for undesirable behavior & 1.667 & 3.407 & .813 & 11.126 & $.000 *$ \\
\hline Avoidance of information overload & 1.481 & 2.665 & 1.001 & 6.150 & $.000 *$ \\
\hline Consistency & 1.593 & 2.926 & .920 & 7.532 & $.000 *$ \\
\hline Play/leisure for the child & 1.296 & 3.074 & .847 & 10.902 & $.000 *$ \\
\hline Moving on to another behavior & 1.926 & 2.148 & .934 & 1.237 & .227 \\
\hline
\end{tabular}

$\mathrm{N}=27 ;$ Degree of freedom $=26 ; *$ significant; $\mathrm{T}(\mathrm{tab})=2.056$ 
Nigg,( 2001) report that lack of parenting skills for the parents of children with ADHD, lower their sense of parenting competence and lead to less satisfaction as parents. Kazdin and Whitley (2003) found that the stress of the parent influences parent disciplinary practices which directly promotes and escalates aggressive and oppositional child behavior. When the parents are tensed up, they tend to have a higher level of irritability and pay more attention to the deviant behavior and thereby negatively influence increased deviant behavior. As a result of this, some parents might simply give up with frustration and increased distress.

\section{Implications for Practice}

Setting up a behavior modification program provides the structure that children with ADHD often need to succeed and could also be of a tremendous benefit to the children with ADHD within the school settings. This is because effective programs benefit from home and school cooperation. The training ought to commence as soon as the parent is informed of the child's ADHD condition and begins treatment. Also, ADHD children need to be educated from the onset of diagnosis regarding how to cooperate with their parents. They need to be taught effective communication and mediation skills when conflict arises. This becomes necessary because of the stress and strain the parents pass through in raising ADHD children. Parenting ADHD children without relevant training has been found to lead to financial burden (Swensen et al., 2003), and increased alcohol consumption (Chronis et al., 2003; Pelham and Lang, 1999).

Techniques for use in schools include token economies, class rules, and attention to positive behavior, as well as time-out and response cost programs. Reinforcements may be dispensed through the use of daily report cards. The homework notebook is useful in improving completion of assignments.

According to Giler (2000) the reward technique works well with most children, although children with ADHD may need more frequent rewards but the system of rewards and penalties should be well managed for it to be effective modifying a child's behavior.

Children with ADHD can find learning a difficult, frustrating and painful process. Because their grades often seem lower than those earned by other children they may not see a relationship between effort expended in school and academic success. Hence, motivating them to achieve academically can be especially challenging.

Community resources need to be in place from the onset of learning to deal with raising an ADHD child, in order for parents to have access to a support service when conflict and difficult times occur.

Multimodal treatment might be more useful in helping the children that have ADHD. This becomes necessary because in addition to the specific training in parenting skills, the need to address multiple problems favors multimodal treatment of these children. In the clinical setting, multimodal treatments may be indicated to address ADHD target symptoms that are not sufficiently improved by medication alone. The combination of medication, classroom behavior therapy with family therapy might be more effective. Supportive therapy may be useful for children who do not have satisfying relationships with adults either because parents are unavailable or unsuitable.

Cooperation between the school and home is very important because close cooperation between parents, peers, school, and community will foster peace and peaceful living for the ADHD children and their significant others. Parents can help in the guidance role by helping with time management, getting a weekly report from the school, and discussing school issues regularly with their children with ADHD.

More research would be needed on this topic with more participants and in different locations before generalizations could be inferred. Also, researchers could carry out more works regarding the effect of parenting skills training on the academic performance of their ADHD children.

The policy-makers would need to pay special attention to the findings of relevant researches and consider the implementation relevant policies.

\section{REFERENCES}

American Psychiatric Association 1994. Diagnostic and Statistical Manual of Mental Disorders: DSM-IV. ( $4^{\text {th }}$ Ed). Washington D.C.: American Psychiatric Association.

Barkley, R.A. 1990. Attention-Deficit Hyperactivity Disorder: A Handbook for Diagnosis and Treatment. New York: The Guildford Press.

Barkley, R.A. 2000. Taking Charge of ADHD: The 
Complete, Authoritative Guide for Parents. New York: The Guildford Press.

Biederman J, S. V. Faraone, K. Keenan, D. Knee and M. F. Tsuang. 1990. "Family-Genetic and Psychosocial Risk Factors in DSM-III Attention Deficit Disorder." Journal of the American Academy of Child and Adolescent Psychiatry, 29(4): 526-533.

Chronis, A.M., B. B. Lahey, W. E. Phelham, H. L. Kipp, B. L. Baumann and S. S. Lee 2003. "Psychopathology and Substance Abuse in Parents of Young Children with ADHD." American Academy of Child and Adolescent Psychiatry, 42(12): 1424-1432.

Consensus Development Panel.. 1982. Defined Diets and Childhood Hyperactivity. National Institutes of Health Consensus Development (NIHCD) Conference Summary, 4(3): 5-8

Giler, J.Z. 2000. Socially Adept: A Manual for Parents of Children with ADHD and/or Learning Disabilities. Santa Barbara: CES Publications.

Hoover D.W. and R. Milich. 1994. "Effects of Sugar Ingestion Expectancies on Mother-Child Interaction." Journal of Abnormal Child Psychology, 22: 501-515.

Kazdin, A.E., and M. K. Whitley. 2003. "Treatment of Parental Stress to Enhance Therapeutic Change Among Children Referred for Aggressive and Antisocial Behavior." Journal of Consulting and Clinical Psychology, 71(3): 504-515.

National Health and Medical Research Council (NHMRC) 1997. Attention Deficit Hyperactivity Disorder. Australia, National Health and Medical Research Council.

Oliner, P., S. Oliner and M. B. Gruber.1991. "Altruism and Peace: Propositions based on Gender and CrossCultural Comparisons." International Journal of World Peace 37

Pelham, W.E. and A. R. Lang. 1999. "Can Your Children Drive You to Drink?" Alcohol Research and Health 23 (4), 292-298. Retrieved November, 12, 2005 from http://www.athealth.com/consumer/disorders/ adhdparents.html

Podolski, C. and J. T. Nigg. 2001. "Parent Stress and Coping in Relation to Child ADHD Severity and Associated Child Disruptive Behavior Problems." Journal of Clinical Psychology. 30(4): 503-513.

Silver, L.B. 1999. Attention-Deficit/Hyperactivity Disorder: A Clinical Guide to Diagnosis and Treatment for Health and Mental Health Professionals. $2^{\text {nd }}$ Ed. Washington D.C.: American Psychiatric Press, Inc.

Swensen, A. R., H. G. Birnbaum, K. Secnik, M. Marynchenko, P. Greenberg, and A. Claxton. 2003. "ADHD: Increased Costs for Parents and their Families." American Academy of Child and Adolescent Psychiatry. 42(12): 1415-1423.

United Nations Educational Scientific and Cultural Organizations (UNESCO) 1999. Manifesto 2000 for the Culture of Peace and non-violence Retrieved November, 12, 2005 from http://www.unesco.org/ manifesto2000

The ADHD Molecular Genetics Network. 2002. "Report from the third international meeting of the attention-deficit hyperactivity disorder molecular genetics network." American Journal of Medical Genetics, 114: 272-277.

Weinberg, H.A. 1999. "Parent Training for ADHD: Parental and Child Outcome." Journal of Clinical Psychology, 55(7): 907-913.

Wender, P. H. 2002. ADHD: Attention-Deficit Hyperactivity Disorder in Children and Adults. Oxford: Oxford University Press.

Wodrich, D. L. 2000. Attention-Deficit/Hyperactivity Disorder: What every Parent wants to Know. $2^{\text {nd }}$ Ed. Baltimore: Paul H. Brookes Publishing Co., Inc.

Wolraich, M., R. Milich, P. Stumbo, F. Schultz. 1985. “ The Effects of Sucrose Ingestion on the Behavior of Hyperactive Boys.” Pediatrics, 106: 657-682. 